\title{
ARE MARTENSITIC PHASE TRANSITIONS IN PURE GROUP 3 AND 4 METALS DRIVEN BY LATTICE VIBRATIONS?
}

\author{
A. HEIMING*, W. PETRY*, and J. TRAMPENAU*** \\ *Institut Laue-Langevin, 156X, 38042 Grenoble cedex France \\ **Institut für Metallforschung, Universität Münster, 4400 Münster, Germany
}

\begin{abstract}
The phonon dispersion in the high temperature bcc phase of the group 3 and 4 metals $\mathrm{Sc}, \mathrm{La}, \mathrm{Ti}, \mathrm{Zr}$ and $\mathrm{Hf}$ have been determined by inelastic neutron scattering. In these pure elements the lattice vibrations are characterized by a valley of low energy modes, which are extremely damped, i.e. lifetimes as short as one vibrational period are measured. It is shown that these low energy vibrations exhibit a particular temperature behavior and are directly related to the transformation into the low temperature hcp or fcc phase or into the hexagonal $\omega$-phase under increasing pressure.
\end{abstract}

\section{Introduction}

Over the last years considerable interests have been focused on an understanding of the role of lattice vibrations as a driving force for undergoing martensitic phase transitions. The most suitable systems to study are pure elements $/ 1 /$, which can be treated theoretically even from first principles (see for example $/ 2,3,4 /$ ). However, due to experimental problems mostly complex alloy systems have been investigated so far (for examples see $/ 5 /$ ).

In this paper we report on temperature dependent measurements of the phonon dispersion in the bcc phase of the pure elements $\mathrm{Sc}, \mathrm{La}, \mathrm{Ti}, \mathrm{Zr}$ and Hf. On lowering the temperature the rather open bcc structure of these metals transforms martensitically to the closed packed hcp structure (for La a transition to the fcc structure occurs at $865^{\circ} \mathrm{C}$.). We want to show, how the elongations of particular lattice vibrations are related to the martensitic transitions from the high temperature bcc phase either into the low temperature hcp (or fcc) phase or under increased pressure into the hexagonal $\omega$-phase.

\section{Experimental}

The experimental difficulty in studying the phonon dispersion of the group 3 and 4 metals in their high temperature bcc phase lies in the occurrence of the martensitic phase transition. A bcc single crystal needed for the inelastic neutron scattering investigation is destroyed into many 
grains of the low temperature phase, as soon as the respective transition temperature is reached (for example $882^{\circ} \mathrm{C}$ for $\mathrm{Ti}$ ). In order to overcome this experimental problem we have constructed a special single crystal growth and measuring furnace, which allows to grow metallic single crystals by the zone melting technique in situ on the neutron spectrometer and perform measurements on the same crystal without any intermediate cooling. Single crystals of good quality and typically $40 \mathrm{~mm}$ length and $10 \mathrm{~mm}$ in diameter were obtained. The measurements were performed on the 3 -axis spectrometer IN3 and IN8 at the high flux neutron reactor of the Institut Laue-Langevin in Grenoble. Where possible, temperature dependent studies were performed over the whole range of existence of the bcc phase.

\section{Results and Discussion}

Exemplarily for the various elements investigated the phonon dispersion in bcc-Hf / $6 /$ is shown in fig. 1. All characteristic features for the phonon dispersions in the group 3 and 4 metals car be described within this example:

1. The longitudinal $L[\xi \xi \xi]$ phonon branch exhibit an extreme dip at $\xi=2 / 3$. It turns out, that this particular phonon is strongly damped and phonon intensities can be found even at zero energy transfer.

2. The transverse $T_{1}[\xi \xi 0]$ branch with $[\overline{1} 10]$ polarization is of exeptionally low energy. The phonon frequencies of this branch show a remarkable temperature dependence, i.e. approaching the transition temperature, this branch softens considerably.

3. The transverse $T_{1}[\xi \xi 2 \xi]$ branch is also of low energy and extremely damped. It can be shown by geometrical considerations, that in the transverse $\mathrm{T}_{1}[\xi \xi 2 \xi]$ branch the $\xi=1 / 3$ phonon is identical to the $\mathrm{L} 2 / 3(111)$ phonon and that the $\xi=1 / 2$ phonon is identical to the $\mathrm{T}_{1} 1 / 2(110)$ phonon. This implies, that the two above mentioned particularities are directly related to each other and do not form singularities!

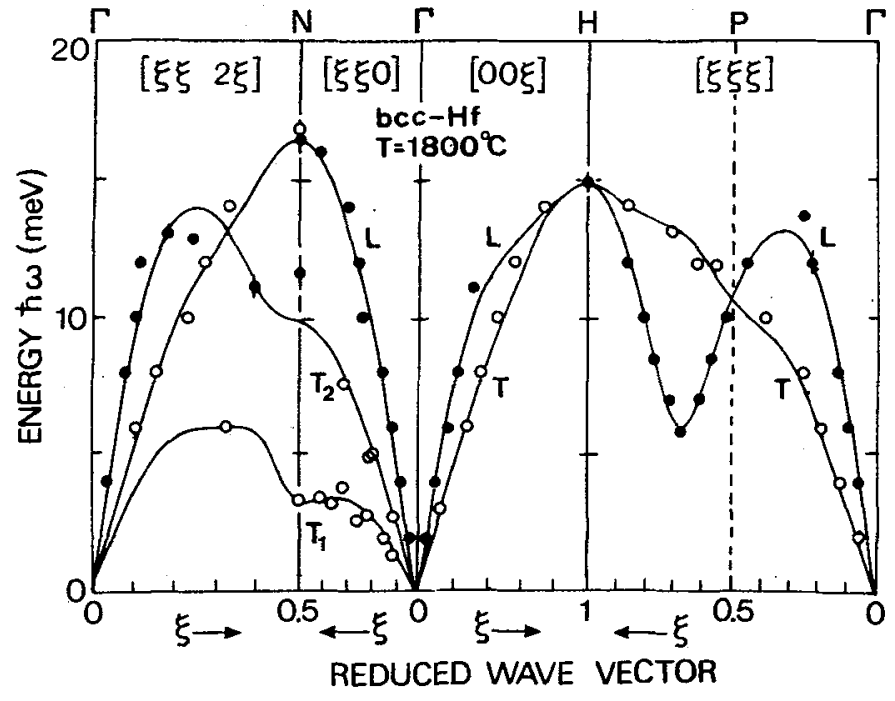

Fig. 1.: Phonon dispersion of bcc-Hf measured at $1800^{\circ} \mathrm{C}$ /6/. All measured dispersions in the various elements can be described by a Bornvon Kármán fit with force constants up to the fifth neigbour shell (full line). 
The details of the phonon dispersion of the other elements are reported elsewhere (Sc $/ 7 /, \mathrm{La}$ $/ 8 /, \mathrm{Ti} / 9 /$ and $\mathrm{Zr} / 10 /)$.

\section{a) The bcc to $\omega$ transformation}

The $\omega$-phase has been identified in all group 4 metals under high pressure and is found in several alloys of the group 4 metals as precipitation at room temperature $/ 11 /$. The crystallographic relationship between the bcc and $\omega$ phase reads:

$$
(111)_{b c c} / /(0001)_{\omega} \text { and }[\overline{1} 01]_{b c c} / /[01 \overline{1} 0]_{\omega}
$$

The bcc to $\omega$ transformation is obtained when two neighbouring (111) planes of the mother bcc phase move towards each other, whereas every third stays at rest (fig. 2). The hexagonal $\omega$-phase is obtained, when the two (111) plane collapse.

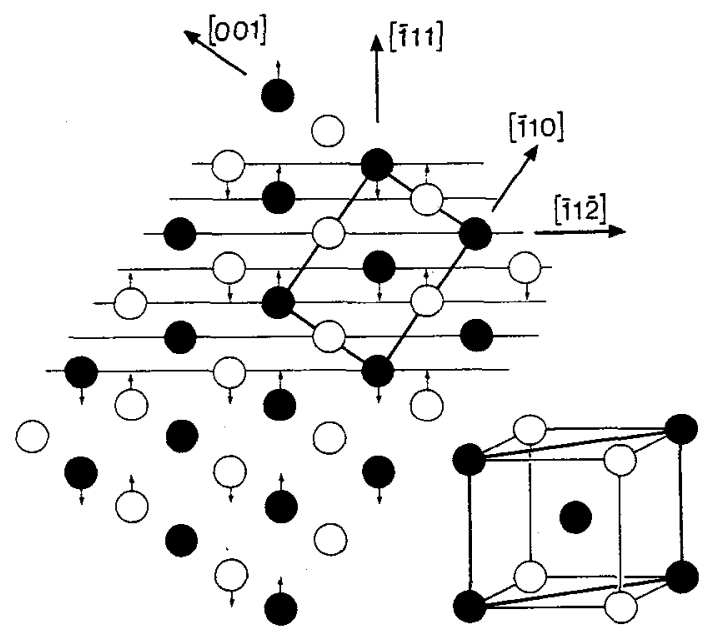

Fig. 2.: Displacements of atoms in a (110) plane due to the $L 2 / 3(\overline{1} 11)$ phonon. Within chains of nearest-neigbour atoms along [111] the atoms do not alter their distance. Solid circle: atoms in the (110) plane; open circles: atoms in the (110) plane below and above.

Dynamically the transformation is achieved by the L2/3(111) phonon (see fig. 2). Extensive investigations of this phonon in the various elements of the group 3 and 4 metals show, that it can be described within the framework of an overdamped oscillator $/ 9 /$. For example, in bcc-Ti a phonon energy of $\hbar \omega=11 \mathrm{meV}$ and a damping of $\Gamma=15 \mathrm{meV}$ were obtained. This strong damping causes that inelastic intensity can be found even at zero energy transfer. It should be pointed out that beyond the intensity of inelastic origin no further elastic intensity is found. This clearly reveals that no stable embryos of the $\omega$-phase in the bcc phase of the pure elements exist. Furthermore we could not detect any temperature dependence of the phonon energy $\hbar \omega$ of this mode in the investigated metals. This is in agreement with the $\mathrm{p}-\mathrm{T}$ phase diagram of the group 4 metals. In these elements the $\omega$-phase can only be obtained under high pressure $/ 11 /$.

From the experimental fact that the L2/3(111) does almost not change over the whole range of temperature where the bcc structure is stable for the various elements we conclude that the weakness towards $\omega$-fluctuations is an intrinsic bcc property. This view is supported by geometrical and electronic arguments. i) The L2/3(111) phonon is the only phonon, which moves chains of atoms in [111] direction without altering the nearest-neigbour distance between atoms within these chains. Since strongest forces are expected to apply between nearest-neigbour atoms, they do not contribute to this particular phonon mode $/ 9 /$, thus leading to low phonon energies. 
This purely geometrical effect applies to all metals with bcc structure and the difference between the various elements with bcc structure is due to the electronic structure. ii) First principle frozen phonon calculations / 2 / show that in the group 4 metals the valence charge density is concentrated in $\mathrm{d}$-bonds along chains in [111] direction with strong interaction along a chain but with little interaction between neighbouring chains. Thus the purely geometrical effect is modulated by the particular electronic structure. Filling up the d-band by going from group 4 to group 6 metals in the periodic table reveals that the $d$-bonds entangle the [111] chains and give rise to extra forces which oppose the shearing motion between neigbouring [111] chains resulting in an increase of the phonon energy.

\section{b) The bcc to hep transformation}

This transformation occurs on lowering the temperature in $\mathrm{Ti}\left(883^{\circ} \mathrm{C}\right), \mathrm{Zr}\left(862^{\circ} \mathrm{C}\right), \mathrm{Hf}$ $\left(1742^{\circ} \mathrm{C}\right)$ and $\mathrm{Sc}\left(1337^{\circ} \mathrm{C}\right)$. The crystallographic relation between the bcc and hcp structures was first established by Burgers /12/

$$
(110)_{\mathrm{bcc}} / /(0001)_{\mathrm{hcp}} \text { and }[\overline{1} 11]_{\mathrm{bcc}} / /[\overline{2} 110]_{\mathrm{h} c \mathrm{p}}
$$

The transformation can be achieved by the superposition of two lattice vibrations: i) The transverse $T_{1} 1 / 2(110)$ phonon with $[\overline{1} 10]$ polarization displaces neighbouring (110) planes in [110] direction. An amplitude of $\delta=\mathrm{a} \cdot \sqrt{2} / 12$ yields the correct stacking sequence of the hcp phase. ii) Two equivalent long wavelength shears such as the (1i2)[i11] and (i12)[1i1] squeeze the bcc octahedron to a regular hcp one, thereby changing the angle from $109.5^{\circ}$ to $120^{\circ}$ in the basal plane. These shears are approximately given by the initial slope of the transverse $T_{1}[\xi \xi 2 \xi]$ phonon branch.
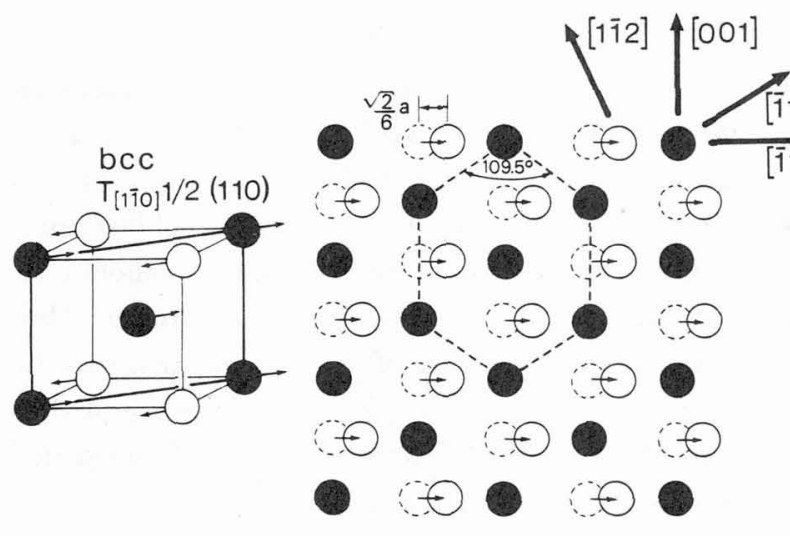

Fig. 3.: Scheme of the bcc to hep transition.

Extensive temperature dependent studies of the transverse $T_{1}[\xi \xi 0]$ phonon branch show a lowering of phonon energies on lowering the temperature towards the transition temperature. For example in $\mathrm{Zr}$ a temperature dependence of $0.34(4) \mathrm{meV} / 100 \mathrm{~K}$ is found for the phonon energy. This softening is of particular importance since on lowering the temperature the lattice usually stiffens and thus an increase of the phonon energy is expected. 
At the transition temperature, the $T_{1} 1 / 2(110)$ phonon of the various metals is of finite energy. Thus the phase transition differs considerably from second order transitions in ferroelectrica, where $\hbar \omega=0$ is observed at the transition temperature. In the bcc phase of the group 3 and 4 metals phonons of the low energy $T_{1}[\xi \xi 0]$ branch are also strongly damped, thus yielding again inelastic intensity down to zero energy transfer. Even though the bcc to hep transformation is temperature induced, elastic intensity scattered by seeds of the low temperature phase on lowering the temperature could not be detected either. On the contrary alloying with small amount of gaseous defects such as oxygen causes the existence of a two phase region (bcc and hcp) over a limited range in temperature. In this case both phases can be observed simultaneously $/ 10 /$.

\section{c) The bcc to fcc transition}

La undergoes a bcc to fcc transformation at $865^{\circ} \mathrm{C}$. Even though this transformation leads also to a closed packed structure, the transformation scheme is different from the one to the hcp structure. Whereas for the bcc-hcp transition the stacking sequence of (110) planes changes from one $A B A B$... to another $A B A B$... sequence, the transition to fcc changes the sequence of (110) planes to ABCABC... . Contrary to the bcc-hcp transition, this transformation can only be achieved by a long wavelength shear. For the bcc to fcc transition these are the (110)[110] shears given by the initial slope of the $\mathrm{T}_{1}[\xi \xi 0]$ phonon branch which correspond to the elastic constant $c^{\prime}=1 / 2\left(c_{11}-c_{12}\right)$. Furthermore the bcc octahedron has to be squeezed to a regular one. This can be achieved by the same two equivalent long wavelength shears - namely (1ĩ2)[111] and (i12) $[1 \overline{1} 1]$ - which were needed for the bcc-hcp transition. The crystallographic relation between bce and fcc thus reads

$$
(110)_{b c c} / /(111)_{f c c} \text { and }[\overline{1} 11]_{b c c} / /[101]_{f c c}
$$

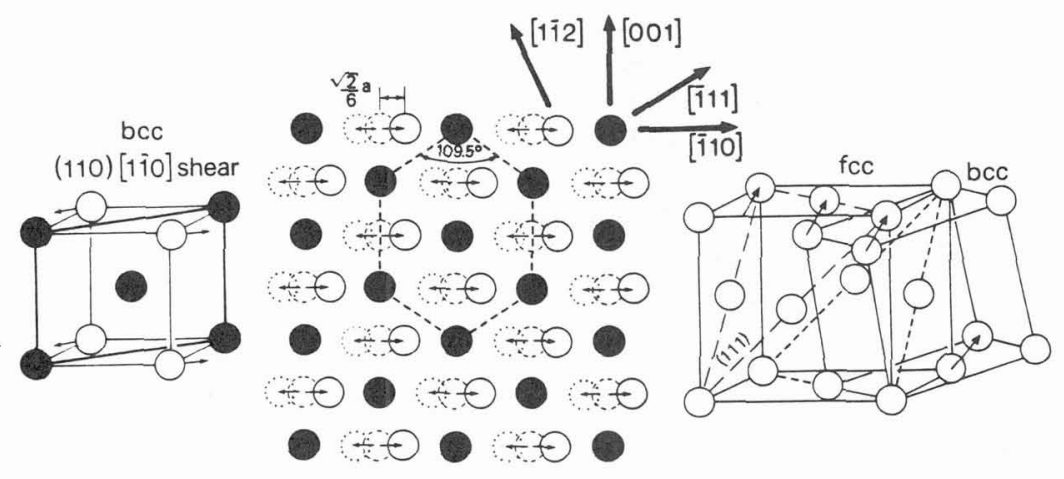

Fig. 4.: Scheme of the bcc to fcc transition.

\section{What stabilizes the bcc structure ?}

First principle frozen phonon calculations for the $\mathrm{T}_{1} 1 / 2(110)$ phonon in $\mathrm{Zr} / 2 /$ showed that at $0 \mathrm{~K}$ the bcc lattice is completely unstable against displacements towards the hep phase and only imaginary phonon energies are obtained. However, including anharmonic effects at finite temperatures, a low, but finite phonon energy is calculated and thereby the bcc structure is stabilized. 
In terms of thermodynamical considerations we calculated the lattice entropy for all investigated elements $/ 6,9,10 /$. High lattice entropies are found caused by the low energy phonons. A comparison of the known excess entropy with the change of the vibrational entropy at the phase transition for $\mathrm{La}, \mathrm{Ti}$ and $\mathrm{Zr}$ shows that the bec structure is stabilized by the vibrational part of the entropy. This makes them different from group 5 and 6 metals where low lying phonon branches are absent and where bcc is the electronically stabilized structure.

Already Zener /13/ proposed that the excess entropy at the phase transition originates from soft acoustic shear modes, i.e. long wavelength modes. Contrarily to Zener we have shown that the modes stabilizing the bcc structure are mainly short wavelength or Brillouinzone boundary phonons. Thereby we confirm a qualitative picture of Friedel / 14/ who discussed in this context the importance of short wavelength modes.

\section{Acknowledgments}

Financial support given by the German Bundesministerium für Forschung und Technologie under Project No. 03-ST1SPH-3 and 03-HE2MUE-0 is greatfully acknowledged. We appreciated the collaboration with C. Herzig.

\section{References}

/1/ W. Petry, Phase Transitions, Vol. 31 (1991) 119

/2/ K.-M. Ho, C.-L. Fu and B.N. Harmon, Phys. Rev. B 28 (1983) 6687; Phys. Rev. B 29 (1984) 1575; Phys. Rev. B 31 (1985) 6775; Phys. Rev. Lett. 58 (1987) 1769; Phys. Rev. B 37 (1988) 283

/3/ J.A. Krumhansl and R.J. Gooding, Phys. Rev. B 39 (1989) 3047

/4/ P.A. Lindgard and O.G. Mouritsen, Phys. Rev. B 41 (1990) 688

/5/ The Martensitic Transformation in Science and Technology, edt. E. Hornbogen and N. Jost, DGM Informationsgesellschaft Verlag 1989

/6/ J. Trampenau, A. Heiming, W. Petry, M. Alba, C. Herzig, W. Miekeley and H. R. Schober, Phys. Rev. B 43 (1991) 10963

/7/ W. Petry, J. Trampenau and A. Heiming, to be published

/8/ F. Güthoff, A. Heiming, W. Petry, C. Stassis and J. Trampenau, to be published

/9/ W. Petry, A. Heiming, J. Trampenau, M. Alba, C. Herzig, H.R. Schober and G. Vogl, Phys. Rev. B 43 (1991) 10933;

/10/ A. Heiming, W. Petry, J. Trampenau, M. Alba, Chr. Herzig, H. R. Schober and G. Vogl, Phys. Rev. B 43 (1991) 10948

/11/ S. K. Sikka, Y. K. Vohra and R. Chidambaram, Prog. Mat. Science 27 (1982) 245

/12/ W.G. Burgers, Physica I (1934) 561

/13/ C. Zener, Phys. Rev. 71 91947) 846

/14/ J. Friedel, J. Physique-Lettres 35 (1974) L-59 